\title{
Contribution à l'archéologie de la distinction sexe - genre
}

\author{
Ce que la psychiatrisation des homosexualités \\ au XIX siècle a fait au sexe
}

Julie Mazaleigue-Labaste

\begin{abstract}
[Résumé] La généalogie de la distinction contemporaine entre sexe biologique, genre et orientation sexuelle remonte le plus souvent à la clinique du transsexualisme à partir des années 1960 aux États-Unis, avant sa formulation dans les théories féministes du genre au cours des années 1970. Mais cette distinction puise en réalité ses sources dans une série de discours scientifiques et médicaux sur les relations de même sexe entre 1850 et le début du $X X^{\mathrm{e}}$ siècle. Ces derniers poursuivaient des objectifs sociaux et politiques : combattre la stigmatisation et la répression des personnes impliquées dans des relations homosexuelles. Ils ont par-là conduit à rompre avec l'appréhension binaire de la sexualité issue des Lumières et ils ont marqué une transformation anthropologique dans la manière de se représenter la sexualité.

Mots-clés : Sexe, Genre, Orientation sexuelle, Politique, Homosexualités, Histoire de la psychiatrie, Histoire de la sexologie, Perversion.
\end{abstract}

[Abstract] The genealogy of the distinction between biological sex, gender and sexual orientation often dates back to the clinic of transsexualism in the U.S.A. during the sixties, before its formulation by the feminist theories in the seventies. But this distinction actually finds its origin in a range of scientific and medical discourses about same sex-relations between 1850 and the beginning of the twentieth century. The latters pursued social and political objectives: fighting against the stigmatization and the repression of the individuals involved in same-sex relations. These discourses have led to breaking with the binary apprehension of sexuality from the Enlightenment, and they have marked an anthropological mutation in the way of representing sexuality.

Keywords : Sex, Gender, Sexual orientation, Politics, Homosexualities,-History of Psychiatry, History of Sexology, Sexual perversion.

On sait que la distinction épistémologique entre les concepts d'orientation sexuelle « choix » d'objet sexuel, conduisant à définir un individu comme homosexuel, hétérosexuel ou bisexuel -, de sexe biologique et de genre - le genre comme construction sociale et culturelle de la masculinité et de la féminité définissant des identités sociales « homme » et «femme » distinctes du sexe biologique et hiérarchisant les rapports sociaux, tel qu'il a pu être premièrement théorisé par Ann Oakley (Oakley, 1972) -, élaborée dans le champ des études féministes et des sciences sociales anglo-saxonnes à partir des années 1970, puise ses sources historiques dans l'étude des relations entre homosexualité, «transsexualisme » et intersexualité au prisme des découvertes endo- 
crinologiques dans les premières décennies du $\mathrm{XX}^{\mathrm{e}}$ siècle et à la clinique du transsexualisme aux États-Unis à partir des années 1950 avec le sexologue John Money, puis le psychanalyste Robert Stoller (Löwy, 2003 ; Castel, 2003, 23-67) ${ }^{1}$. Avant d'être défini comme une construction sociale, le genre a ainsi été distingué du sexe biologique et défini par la sexologie et les savoirs psychologiques en référence à une «identité de genre» de type psychologique, à savoir le sentiment pour une personne d'appartenir à tel ou tel sexe. La présente étude souhaite, dans une perspective d'épistémologie et d'histoire des sciences médicales et psychologiques, mettre l'accent sur une source antérieure de cette distinction entre sexe, genre et orientation sexuelle : l'intersection entre les premiers savoirs sexologiques au XIX ${ }^{\mathrm{e}}$ siècle (Crozier, 2003) qui ont d'abord pris la forme d'une psychologie de la sexualité et l'émergence de politiques de l'identité chez les militants homosexuels masculins. Les discours savants sur les homosexualités et les débats autour de leur statut de "pathologies psychosexuelles » (de perversions) entre 1850 et le début du XX ${ }^{\mathrm{e}}$ siècle ont en effet largement contribué à rompre avec l'appréhension binaire de la sexualité issue des Lumières, c'est-à-dire avec l'idée que le désir sexuel correspond naturellement à la sexuation et doit s'adresser au sexe opposé à celui de l'individu. Trois moments jalonnent cette histoire du début du XIX ${ }^{\mathrm{e}}$ siècle au tournant du XX $\mathrm{XX}^{\mathrm{e}}$ siècle. Le premier (1800-1852) met en scène l'émergence en France de la psychopathologie de la sexualité (le savoir psychiatrique des perversions). Il a abouti à la remise en question du cadre naturaliste traditionnel qui ordonnait la pensée des relations entre sexe et sexualité à travers de nouvelles définitions psychologiques de l'identité des individus impliqués dans des relations de même sexe - et en premier lieu des relations entre hommes. Le second moment de cette histoire prend place en Allemagne, entre 1860 et 1890, dans le contexte de la lutte politique contre la pénalisation des relations homosexuelles. De nouvelles théories psychologiques de l'identité homosexuelle ont alors conduit à la nécessité de dépasser toute logique binaire d'appréhension de la sexualité. Enfin, entre 1890 et 1910, une distinction conceptuelle entre sexe biologique, identité (psychologique et sociale) masculine ou féminine, et choix d’objet sexuel (homo- ou hétéro-sexualité) a été formalisée sous la plume des militants sexologues, et en particulier en France celle de Marc-André Raffalovich.

\section{Généalogie de l'« inversion sexuelle » et logique binaire de la sexualité (1800 - 1852)}

Le premier moment de cette histoire est la généalogie des définitions psychologiques de «l'inversion sexuelle » (qui fut la matrice des concepts d'homosexualité, de

${ }^{1}$ Voir également l'article de Michal Raz dans ce numéro (NdE). 
transvestisme et de transsexualisme) dans la médecine, la psychiatrie et les premières sciences humaines (les "sciences morales»). Dans les premières décennies du XIX siècle, la représentation savante soudait l'objet du désir à la reproduction et donc au sexe biologique. La définition de « sexualité » qui prévalait alors était révélatrice de ce schème de pensée. Naturaliste et linnéenne, elle s'inscrivait dans la continuité des Lumières : le mot « sexualité » désignait ce qui relève de la sexuation et des organes de reproduction, des plantes à l'être humain (Nysten, Littré, Robin 1865, 1381). La perception savante des érotismes s'inscrivait plus largement dans un cadre de représentation pour lequel la nature jouait comme référence éthique (Vidal, 2004) : elle fondait la norme du bien sexuel défini par le coït hétérosexuel modéré dans le cadre matrimonial (Corbin 2007, 55) et par une économie de la gestion de soi s'exprimant dans une éthique de la modération (Muchembled, 2005, 159). Le problème central était alors celui du contrôle des excès sexuels affaiblissant l'organisme et l'esprit. Certes, l'existence de «déviations sexuelles» était reconnue. Ces «abus» englobaient les " contre-nature » de la théologie morale moderne (onanisme, bestialité, sodomie qui englobait relations entre personnes de même sexe et parfois onanisme $)^{2}$. Cependant, les types sexuels déviants (onaniste, sodomite, tribade, pédéraste) étaient rapportés au problème de l'excès (Corbin, 2007, 189), à travers le paradigme issu des Lumières qu'était le masturbateur de Tissot (Tissot, 1991[1761] ; Singy, 2014). Pour la pensée savante, pédérastes et tribades partageaient ainsi le destin de tou.te.s les « excessives.fs sexuel-le·s », débauché·e·s ou onanistes : l'épuisement vital (Virey, 1818, 144-145). Parce qu'elles étaient rapportées à la question de la modération, les spécificités sexuelles ne pouvaient donc pas être décrites de manière autonome. Toutefois, hors de la pensée scientifique et médicale, des portraits esquissant des identités non assimilables au sexe et relevant des stéréotypes socio-sexuels émergeaient. Depuis la fin du XVIII siècle, les types et les portraits du pédéraste, de la tribade, de l'«antiphysique » (l'homme qui aime les hommes) avaient commencé à s'individualiser avec le développement des communautés homosexuelles sous les Lumières et leur quadrillage policier (Ariès 1982, 59 ; Sibalis, 1996, 81 ; Trumbach, 1998 ; Tamagne, 2001, 53-87). Et, dans les premières décennies du XIX ${ }^{\mathrm{e}}$, les taxinomies sociales se multipliaient dans le but de rationaliser les pratiques d'ordre de la nouvelle société (Corbin 2007, 134). Sous la monarchie de Juillet a ainsi émergé en France une "proto-sexologie de commissariat » (Murat, 2007), diffusée notamment grâce aux romans de Balzac. Cette typologie policière insistait sur un personnage particulier, la «tante», dont elle livrait un type

\footnotetext{
${ }^{2}$ La sodomie ne désignait pas cet acte particulier qu'est la pénétration anale, mais était définie en référence à la théologie morale et pouvait inclure la pédérastie (relations sexuelles entre hommes), le tribadisme (entre femmes) et la masturbation (Ariès, 1982, 58 ; Hurteau, 1993, 9-10; Jordan, 2006 pour une histoire de la catégorie de "sodomie " à partir de la théologie morale médiévale).
} 
comportemental ${ }^{3}$. Ces portraits présentaient indéniablement une spécificité qualitative qui les éloignait du schème de l'excès sexuel : celui d'une inversion des stéréotypes socio-sexuels. L'homme désirant les hommes était féminisé, et la femme aimant les femmes masculinisée. Bien que la description de cette inversion intégra parfois des références physiologiques (dans la représentation du pédéraste-tante au corps glabre et féminisé, et de la tribade au clitoris hypertrophié), elle était surtout sociale et comportementale.

Ce premier moment conduisit à l'apparition, entre 1849 et 1852, des premières définitions psychologiques d'une d'identité censément propre aux homosexuel.le.s et distincte du sexe biologique et des rôles sociaux. On peut nommer cette identité «psychosexuelle" sans anachronisme, dans la mesure où elle définit la personne relativement à sa sexualité considérée comme un élément non physique, mais psychologique. Ces définitions ne sauraient toutefois être ressaisies que sur la toile de fond que fut l'invention du concept moderne de "perversion sexuelle», c'est à dire l'apparition des premières théories psychologiques sur les déviances érotiques dans le champ de la psychiatrie. Ainsi, en France, les homosexualités furent psychiatrisées en 1849 par l'aliéniste Claude-François Michéa dans le contexte d'une classification plus large des perversions (de la nécrophilie au sadisme) (Mazaleigue-Labaste, 2014, 137150). Michéa était homosexuel, et cette donnée récemment mise en évidence est déterminante (Révenin 2007, 29 ; Féray 2015). En effet, les théories savantes ici présentées, qui ont proposé des définitions d'une identité psychologique propre aux personnes engagées dans des relations de même sexe, ont été développées par des savants homosexuels. Michéa avait ainsi l'ambition scientifique et politique d'expliquer psychologiquement les attirances homosexuelles afin de les déstigmatiser. À cet effet, il forgea une catégorie de perversion sexuelle : l'« amour grec » comme « amour des sexes semblables » (subdivisé en philopaedie et tribadisme), inné et distingué des pratiques sexuelles occasionnelles entre personnes de même sexe (Michéa, 1849, 339). Cet « amour grec » s'éloignait donc des excès sexuels comme des stéréotypes sociaux : les «tribades » et « antiphysiques » n'étaient plus des «libertins vicieux ». L'analyse se décalait des pratiques au penchant sexuel, c'est-à-dire à la dimension psychologique du désir homosexuel, ce qui conduisit à définir un type psychologique caractérisé par son désir et son identité spécifiques. Trois ans plus tard, en 1852, le médecin légiste allemand Johann Ludwig Casper (lui aussi homosexuel), proposa une théorie psychiatrique très proche qui s'enracinait dans une tradition germanique de réflexion philologique et médicale sur les homosexualités masculines (Casper, 1852 ; Mazaleigue, 2013, 38-39) qu'il définit comme « hermaphrodisme moral »

\footnotetext{
${ }^{3}$ Il sera le point d'appui de la médecine légale de la pédérastie d'Ambroise Tardieu à partir des années 1850, qui en fixera le stéréotype moral et social (Tardieu, 1857/1859).
} 
(Casper, 1862 vol. 1,118). Force est de constater ici qu'en dépit de la prise en compte des relations entre femmes, c'était cependant le désir entre hommes qui focalisait l'attention de ces médecins, parce qu'ils étaient eux-mêmes des hommes impliqués dans des relations de même sexe dans deux espaces juridiques où les relations homosexuelles masculines étaient bien davantage judiciarisées que celles entre femmes ${ }^{4}$.

La conceptualisation de ces identités psychosexuelles définissait ainsi un type de subjectivité psychologique propre aux personnes impliquées dans les relations de même sexe (et non simplement un type d'identité référé aux comportements sociosexuels dans un contexte de stigmatisation, comme pouvaient l'être les stéréotypes de la tante et de la tribade) et distinguée du sexe biologique. Mais elle restait tout de même tributaire de l'articulation entre sexualité et sexuation. Car le modèle de l'hermaphrodisme psychologique présent chez Michéa et Casper gardait pour référence implicite la binarité biologique des sexes - c'est pourquoi Michéa postulait la présence d'un organe analogue à l'utérus chez les hommes philopèdes. Celui ou celle qui désire son semblable devait nécessairement avoir quelque chose de l'autre sexe. Pour cette raison, les Scythes antiques, dont la tradition historique soulignait que certains vivaient "comme des femmes", c'est-à-dire adoptaient des habitus comportementaux féminins, étaient pour Michéa tout autant philopèdes que les hommes désirant les hommes (Michéa, 1849, 338-339). Le schéma naturaliste binaire faisait encore obstacle à la séparation entre identité psychologiquement et/ou socialement assumée par l'individu (homme ou femme) et orientation sexuelle.

\section{La fragilisation du modèle binaire : l'« inversion sexuelle » dans le premier militantisme homosexuel germanique et ses diffusions (1860 - 1890)}

À partir des années 1860, les relations de même sexe, et plus spécifiquement entre hommes, gagnèrent en importance dans l'espace scientifique et médical ouesteuropéen, et le modèle soudant sexualité et sexuation fut fragilisé par des innovations dans l'explication psychologique des homosexualités. Mais l'origine de ces transformations conceptuelles ne fut pas la psychiatrie, qui restait tributaire des théories du milieu du siècle faisant du désir homosexuel une maladie mentale. Et leur creuset ne fut pas la France, mais l'espace germanique. Les homosexualités devinrent en effet un objet important des sciences médicales et psychologiques d'abord en Allemagne et en

\footnotetext{
${ }^{4} \mathrm{Si}$, en France, la répression passait par la police et non d'abord par la Justice pénale puisque les relations de même sexe avaient été dépénalisées en 1791, elles étaient fortement réprimées par la loi dans l'espace germanique.
} 
Autriche-Hongrie en raison des coordonnées spécifiques du contexte social, juridique et politique dans lesquelles étaient prises les relations de même sexe. Un nouveau concept, l'«inversion sexuelle», fut forgé dans le cadre de luttes militantes à l'encontre de la pénalisation de ces relations. Il désigna d'abord une identité psychologique propre aux homosexuel-le·s, sa vocation étant de réfuter sur des bases théoriques toute forme de pénalisation et de stigmatisation. Par la suite, les psychiatres germaniques puis européens s'en emparèrent pour définir une maladie de la personnalité.

Le contexte pénal très répressif de l'Empire Allemand et de l'Autriche-Hongrie fut déterminant. Les lois y criminalisaient en effet les relations sexuelles entre hommes (et entre hommes et entre femmes pour le code criminel autrichien) (Mazaleigue, 2007). Ces relations étaient alors rendues visibles dans la sphère publique, ce qui formait le terrain d'un militantisme médiatisé et offrait aux médecins l'accès à des observations de cas via leur implication dans le système judiciaire en tant qu'experts ${ }^{5}$ (Oostherhuis, 2000, 38 ; Tamagne, 2001, 91-131). Ainsi, en Allemagne, où les relations entre hommes (mais pas entre femmes) étaient pénalisées, une revendication homosexuelle attaquant la criminalisation de la sodomie émergea dans les années 1860 . Lors du Congrès des juristes de Munich en 1867, le juriste et journaliste homosexuel Karl Ulrichs s'opposa à l'intégration de l'ancien article 143 du code prussien aux nouvelles lois allemandes en préparation. Ce fut sans succès, puisque le $\$ 175$ du Code pénal de l'Empire d'Allemagne de 1871 continua à sanctionner les actes sexuels entre hommes au même titre que ceux avec des animaux. En parallèle de cette contestation publique des lois, la théorie fut aussi un instrument de lutte 6 . À partir de 1864, Ulrichs, très au fait de l'ensemble des théories scientifiques du temps, publia une série d'essais polémiques dans lesquels il mobilisa la psychologie, les nouvelles sciences biologiques et le cadre de pensée darwinien (Brooks, 2012[2010]) pour inventer une nouvelle conception psychologique de l'identité homosexuelle masculine radicalement opposée à la répression pénale (Ulrichs, 1994 [1864-1880]). Mais les enjeux de cette théorie allaient beaucoup plus loin que l'opposition aux lois : elle devait réfuter la psychiatrisation du désir homosexuel (l'idée que ce désir relevait d'une "perversion sexuelle», c'est-à-dire d'une maladie mentale) et plus largement toutes les conceptions stigmatisant les relations de même sexe, et devait aussi fonder l'émancipation des homosexuel-le·s dans la société politique et civile.

Ulrichs définit ainsi un genre naturel d'êtres humains dans une perspective évolutionniste et ontogénétique. Selon lui, la différenciation de l'embryon sexuellement

\footnotetext{
${ }^{5}$ On ne comptait pas de femmes parmi les médecins-légistes au XIX ${ }^{\mathrm{e}}$ siècle.

${ }^{6}$ En Autriche, le journaliste germano-hongrois Karl Maria Kertbeny, proche et correspondant d'Ulrichs, forgea le terme « Homosexualität » en l'opposant à la « Normalsexualität » en 1868.
} 
indéterminé commence par la formation des organes sexuels puis du psychisme, enfin de cette partie du psychisme qu'est l'instinct sexuel. Mais cette évolution n'est pas toujours complète et la population humaine déploie des variations dont certaines abritent un instinct sexuel contraire à leur organisation génitale et physique. Ce sont des êtres psychologiquement hermaphrodites caractérisés par un décalage entre le sexe du corps et celui de l'esprit. Ulrichs applique ce schéma aux femmes comme aux hommes impliqué.e.s dans des relations de même sexe, mais il dédie bien davantage ses analyses à ces derniers (puisque c'étaient eux la première cible de la pénalisation en Allemagne) qu'il nomme les Urnings (" uranistes » en français). S’il n'employait pas l'expression « inversion sexuelle» qui n'apparaîtra qu'en 1878 sous la plume d'Arrigo Tamassia (Tamassia, 1878), Ulrichs faisait néanmoins bien jouer un concept d'inversion sexuelle pour définir une identité psychosexuelle spécifique : l'uranisme, propre aux individus dont l'organisation physique est conforme à leur sexe biologique, mais dont l'instinct sexuel féminin les conduit à être attirés par les hommes ${ }^{7}$.

Toutefois, si cette théorie permettait de séparer les processus de sexuation organique et l'identité psychosexuelle (il peut y avoir une psyché féminine dans un corps d'homme), elle ne permettait pas de scinder cette dernière de l'orientation sexuelle. Car l'usage politique des sciences biologiques par Ulrichs était à double tranchant. Ces dernières faisaient peser des contraintes fortes obligeant à conserver le schéma de l'hermaphrodisme psychique. En effet, dans une perspective évolutionniste, l'humanité est une espèce animale. La sexualité doit donc être adossée d'une manière ou d'une autre à la sexuation : l'instinct sexuel est lié au sexe biologique, parce que sa finalité naturelle est la reproduction de l'espèce et qu'il faut pour ce faire un mâle et une femelle. La structure restait donc dualiste : le mâle humain qui aime ses semblables devait avoir quelque chose de la femelle humaine (c'est le concept même d'«inversion sexuelle »). Ce qui apparaît dans la définition connue de l'uraniste comme "âme de femme dans un corps d'homme " (anima muliebris in corpore virili inclusa) (Ulrichs, 1994 [1864-1880], vol. 2, 509, 553), mais aussi dans l'appropriation des théories d'Ulrichs par la psychiatrie germanique avec l'invention du concept de «sentiment sexuel contraire » (konträre Sexualempfindung) par l'allemand Karl Westphal en 1869 (Westphal, 1969), point de départ d'une inflation textuelle pléthorique sur l'homosexualité comme perversion. Cette catégorie qualifiait des troubles pathologiques de l'identité psychosexuelle qui englobait l'attirance pour des personnes de même sexe, le sentiment d'appartenir à l'autre sexe ou l'habitude d'endosser les attributs vestimentaires et comportementaux féminins pour des hommes et masculins pour des femmes ${ }^{8}$. Elle était ordonnée à la perception massive d'une inversion du

\footnotetext{
${ }^{7}$ Les Dionings sont, eux, des hommes dont l'instinct sexuel est conforme à leur sexe.

${ }^{8}$ Cette catégorie correspond en France à la « sensation croisée de l'individualité sexuelle » (Chatelain, 1871).
} 
« sexe-genre », sans différence de niveau entre orientation sexuelle et habitus sociaux et comportementaux (Chaperon, 2007, 105-107). Le point est évident chez Richard von Krafft-Ebing, le «pape des perversions », pourtant plus proche d'Ulrichs et militant pour l'abolition du \$175 du Code Pénal allemand pénalisant les relations entre hommes : l'inversion se déploie sur une échelle de degrés allant de « l'hermaphroditisme psychique » à la conviction délirante d'appartenir à l'autre sexe accompagnée d'une transformation de l'« habitus physique »" (Krafft-Ebing, 1895, 246247). Quel que soit le degré de leur inversion, la femme qui désire les femmes et l'homme qui désire les hommes gardent encore quelque chose du sexe opposé. Il était dès lors conceptuellement impossible d'isoler un « désir homosexuel ${ }^{10}$.

Si la théorie d’Ulrichs a formé une charnière dans la pensée de la sexualité, ce n'est donc pas tant en raison de ses conclusions que des limites qu'elle a rencontrées. Car elle n'a pas rempli un de ses objectifs, à savoir expliquer la genèse des homosexualités. Puisque chez lui, l'identité psychosexuelle est définie par l'objet de l'instinct en référence à la binarité des sexes (celui qui est attiré par les hommes a une âme de femme), l'amour de deux uranistes est celui de deux psychismes féminins, une sorte de lesbianisme psychique. L'entreprise d'Ulrichs était donc circulaire : elle expliquait l'homosexualité par l'homosexualité. Les limites du modèle de l'inversion sexuelle ont donc conduit à son dépassement théorique. Parler du désir des hommes pour les hommes et des femmes pour les femmes appelait à distinguer sexe biologique, identité de genre et orientation sexuelle.

\section{Les politiques de l'identité virile : vers une logique ternaire de la sexualité (1890 - 1910)}

C'est en France, dans un contexte moins répressif que son pendant d'Outre-Rhin, que s'est, en premier lieu, opérée cette différenciation au tournant du $\mathrm{XX}^{\mathrm{e}}$ siècle sous une plume qui s'opposait à la fois à la psychiatrisation de l'homosexualité et aux thèses d'Ulrichs, pour des raisons d'abord politiques : celle du journaliste, sexologue et militant Marc-André Raffalovich, dont Patrick Cardon a fait le «pionner des gay studies » (Cardon, 2008[1984], 118), et dont l'objectif était l'intégration sociale et politique des homosexuels dans un pays où, certes, l'homosexualité n'était pas criminalisée, mais où elle continuait à faire l'objet d'une répression policière et d'une forte stigma-

\footnotetext{
${ }^{9}$ La metamorphosis sexualis paranoica, matrice du concept de transsexualisme au XX ${ }^{e}$ siècle (Castel, 2003, 23-67).

${ }^{10}$ D'où les multiples tentatives entre 1890 et le début du XX $X^{e}$ siècle pour expliquer les homosexualités par les découvertes physiologiques (et plus spécifiquement l'endocrinologie) et leur mise en relation avec l'intersexualité (Löwy, 2003).
} 
tisation. Raffalovich développa une série d'études dédiées à l'homosexualité qui ne relevait pas de la psychopathologie, mais de la sexologie qui émergeait en parallèle en Angleterre avec Henry Havelock Ellis et en Allemagne avec Magnus Hirschfeld. En d'autres termes, il ne s'agissait pas de traiter l'homosexualité comme une pathologie mentale, mais comme une variété et une variation possible de la sexualité humaine, et de lui dédier dans cette perspective des analyses à la fois psychologiques, comportementales, sociales, voire d'inspiration sociologique. Raffalovich bénéficia, pour ce faire, d'une tribune scientifique de choix entre 1894 à 1910 dans les Archives d'Anthropologie criminelle, publication de l'école d'anthropologie criminelle de Lyon sous l'influence d'Alexandre Lacassagne. Dès leur fondation en 1886, les Archives contribuèrent, en parallèle des grandes revues médicales comme les Annales d'Hygiène publique et de Médecine légale et les Annales médico-psychologiques, à diffuser les concepts et les théories sur la sexualité dans le monde savant français, et furent en particulier le lieu de discussions avec les théories d'origine germanique. Raffalovich, fort de la place accordée à ses textes dans les Archives, put alors à la fois discuter les thèses d'Ulrichs et promouvoir une nouvelle conception non pathologisante des amours masculines.

Car le discours de Raffalovich portait sur les hommes, pour une raison simple : son objectif était l'intégration sociale et politique des homosexuels à travers la promotion d'un modèle de masculinité conforme aux attentes de la Troisième République. Une telle intégration supposait de lutter contre toute répression sociale et policière, et plus généralement toute stigmatisation des homosexuels. Or, les deux stéréotypes stigmatisants principaux renvoyaient à l'effémination : la figure policière et décriée de la tante liée au monde prostitutionnel et criminel (Chevalier, 1893, 175-199; Revenin, 2005) et la figure psychopathologique de l'inverti (l'homme atteint d'inversion sexuelle, possédant une part féminine), toutes deux opposées au valeurs et attentes éthiques, sociales et politiques de la masculinité Belle-Epoque - l'homme moralement régénéré de la Troisième République, sain de corps et d'esprit, maître de lui-même et de sa vie familiale et sociale, et ainsi bon citoyen (Mazaleigue, 2013, 44, 46). Militer en faveur de l'intégration sociale et politique des hommes aimant les hommes supposait donc de promouvoir une conception psychologique et sociale de l'identité homosexuelle virile, donc de réfuter l'existence d'un lien intrinsèque entre homosexualité et féminité. En conséquence, il fallait récuser les théories faisant appel à l'idée d'une « inversion sexuelle » (uranisme y compris) et définir l'homosexualité uniquement en référence au choix d'objet. C'est ce que fit Raffalovich. Il forgea le concept d'« unisexualité » strictement défini par l'orientation du désir sexuel (désir d'un homme pour un autre homme), non par la présence en soi d'une part féminine, concept dont le sens est alors équivalent au sens contemporain d'« homosexualité » (désir pour une personne de même sexe, toutes choses égales par ailleurs) (Raffalovich, 1896). En d'autres termes, la définition de l'unisexualité conduisit à séparer clairement le choix d'objet (personne de même sexe ou de sexe différent) non seulement de 
la sexuation biologique, mais aussi de l'identité psychologique assumée par l'individu et/ou socialement construite (masculin ou féminin, homme ou femme), c'est-à-dire de ce qui sera bien plus tard théorisé sous les différents concepts d'identité de genre dans le champ de la psychologie, puis des sciences sociales. De cette manière, Raffalovich put promouvoir son modèle de subjectivité homosexuelle «ultra-virile» excluant toute féminité et qui s'opposait tout autant à « l'uraniste » d'Ulrichs, au dandy efféminé de l'esthétique décadentiste qu'à la « tante » du marché prostitutionnel. Ce modèle lui permit d'affirmer la normalité des unisexuels et leur parfaite intégration sociale. En Angleterre, à partir de 1883, John Addington Symonds proposa lui aussi un modèle moral et politique de subjectivité homosexuelle s'éloignant de l'inverti efféminé (Symonds, 1975[1883] ; Ellis, 1975[1897]) et conduisant à conceptualiser de manière autonome l'orientation sexuelle.

Le dépassement du cadre binaire et la séparation claire entre le sexe biologique, l'identité psychosexuelle et/ou socio-sexuelle des personnes (homme/femme) et l'orientation sexuelle (le choix d'objet sexuel ne détermine, ni n'est déterminé par l'identité masculine ou féminine de la personne) fut donc directement lié à l'élaboration d'un modèle identitaire de l'homosexualité masculine aux effets politiques et sociaux ambigus. En effet, la volonté d'intégration sociale et la réfutation d'une quelconque pathologie chez les homosexuels impliquait une certaine normalisation que l'on peut nommer "normalisation de genre » : le " bon » homosexuel se devait d'évincer de sa subjectivité et de son existence tout élément psychologique, comportemental ou social considéré comme féminin. En contrepartie, ceci renforçait évidemment la stigmatisation des hommes homosexuels ne correspondant pas à ce modèle de masculinité viril.

L'histoire de la distinction conceptuelle entre le sexe (biologique), le genre et l'orientation sexuelle a donc commencé avant le développement des savoirs biologiques et sexologiques de l'intersexualité et du transsexualisme dans les premières décennies du $\mathrm{XX}^{\mathrm{e}}$ siècle. Elle articule indissolublement le développement des premiers savoirs de la sexualité français puis germaniques au XIX ${ }^{e}$ siècle qui ont d'abord pris la forme d'une psychologie sexuelle (qui fut d'abord une psychopathologie des perversions) et linvestissement de ces savoirs par des homosexuels en lutte. Ces derniers les ont mobilisés pour élaborer des explications psychologiques du désir homosexuel et de nouvelles catégories d'identité psychosexuelle à visée politique déstigmatisante. On peut alors dire que la psychologisation des homosexualités au XIX ${ }^{e}$ siècle a formé une condition essentielle dans la dynamique de séparation entre les notions de sexe, de genre et d'orientation sexuelle. C'est en effet la psychiatrie (et plus particulièrement des psychiatres homosexuels) qui a livré à partir du milieu du XIX ${ }^{\mathrm{e}}$ siècle les premières définitions d'une identité psychologique propre aux personnes engagées dans des relations de même sexe et bien distincte de la sexuation, dans le cadre d'une entreprise plus large de psychiatrisation des déviances érotiques. Par la suite, les premiers militants homosexuels luttant contre la répression et la stigmatisa- 
tion des relations de même sexe (et surtout des relations entre hommes), comme Ulrichs et Raffalovich, ont mobilisé les savoirs psychologiques pour raffiner et transformer ces définitions, les ont dé-pathologisées et ont défini de nouveaux types d'identité et de subjectivité psychosexuelles, de l'inversion sexuelle à l'unisexualité virile. À l'intersection du champ de la sexologie et de la lutte politique, les modulations théoriques inventées par ces militants qui étaient tout autant des savants, les difficultés qu'ils ont rencontrées et les réponses qu'ils y ont apportées ont ainsi largement contribué à une différenciation que le $\mathrm{XX}^{\mathrm{e}}$ siècle n'a cessé d'affiner et au dépassement relatif d'un cadre de pensée binaire traditionnel soudant sexualité et sexuation au profit d'un système ternaire autorisant la description des variations des sexes, des désirs, des identités et des rôles sociaux.

\section{Bibliographie}

Ariès P. (1982), "Réflexions sur l'histoire de l'homosexualité ", in Ariès P., Béjin A. (1982), Sexualités occidentales. Contributions à l'histoire de la sociologie des sexualités, Communications, 35, pp. 56-67.

Brooks R. (2012[2010]), "Transforming Sexuality : The Medical Sources of Karl Heinrich Ulrichs (1825-95) and the Origins of the Theory of Bisexuality », Journal for the History of Medicine and Allied Sciences, 67(2), pp. 177-216.

Cardon P. (2008[1984]), Discours littéraire et scientifique fin-de-siècle. La discussion sur les homosexualités dans la revue Archives d'anthropologie criminelle du Dr Lacassagne (1886-1914). Autour de Marc-André Raffalovich, Paris : Orizons.

Casper J. L. (1852), « Über Nothsucht und Päderastie und deren Ermittelung Seitens des Gerichtsarztes », Vierteljahrsschrift für gerichtliche und öffentliche Medicin, 1, pp. 21-78.

Casper J. L. (1862), Traité pratique de médecine légale, 2 vol., Paris : Baillière.

Castel P-H. (2003), La métamorphose impensable. Essai sur le transsexualisme et l’identité personnelle, Paris : Gallimard.

Chaperon S. (2007), Les Origines de la sexologie. 1850-1900, Paris : Audibert.

Chatelain A. (1871). Compte rendu de « De la sensation croisée de l'individualité sexuelle comme symptôme d'un état psychopathique anormal », Annales médicopsychologiques, série 5, 6, pp. 285-288.

Chevalier J. (1893), L’inversion. Une maladie de la personnalité, Lyon : Storck.

Corbin A. (2007), L'harmonie des plaisirs, Paris : Perrin. 
Crozier I. (2003), « La sexologie et la définition du «normal » entre 1860 et 1900 », in Löwy I., Rouch H. (éd.), « La distinction entre sexe et genre », Cahiers du genre, 34, pp. 17-37, traduction de l'anglais par Oristelle Bonis.

Ellis H. H. (1975[1897]), Sexual inversion, New York: Arno Press.

Féray J-C (2015), L'impossible conciliation ou la vie hérö̈que du Dr Claude-François Michéa, Paris : Quinte-Feuilles.

Hurteau P. (1993), « Catholic Moral discourse on male sodomy and masturbation in the Seventeenth and Eighteenth Centuries », Journal of the History of Sexuality, 4 (1), pp. 1-26.

Jordan M. D. (2007[1998]), L'invention de la sodomie dans la théologie médiévale, Paris : EPEL, traduction par Le Gaufey de Jordan G. (1998), The Invention of Sodomy in Christian Theology, Chicago : University of Chicago Press.

Katz J. N. (2000[1996]), L'invention de l'hétérosexualité, Paris : EPEL, traduction par Michel O. Thévenet (1996), The invention of heterosexuality, New-York: Penguin Books.

Krafft-Ebing R. v. (1895), Psychopathia sexualis, avec des recherches spéciales sur l'inversion sexuelle, Paris: Georges Carré, traduction de la huitième édition allemande par Laurent É., Csapo S.

Löwy I. (2003), « Intersexe et transsexualités : Les technologies de la médecine et la séparation du sexe biologique du sexe social », in Löwy I., Rouch H. (éd.), « La distinction entre sexe et genre », Cahiers du genre, 34, pp. 81-104.

Mazaleigue J. (2007), « Le tribunal comme lieu de savoir et de pouvoir : le rôle de l'expertise médico-légale dans l'émergence de la psychopathologie de la sexualité », La lettre du psychiatre, 3 (8), pp. 169-174.

Mazaleigue-Labaste J. (2013), « De l'amour socratique à l'homosexualité grecque », Romantisme, 159(1), pp. 35-46.

Mazaleigue-Labaste J. (2014), Les déséquilibres de l'amour. La genèse du concept de perversion sexuelle de la Révolution française à Freud, Paris : Ithaque.

Michéa C-F. (1849), « Des déviations maladives de l'appétit vénérien », L’Union médicale, 3 (85), pp. 338-339.

Muchembled R. (2005), L'orgasme et l'Occident, Paris : Seuil.

Murat L. (2007), « La tante, le policier et l'écrivain. Pour une protosexologie de commissariat et de roman », Revue d'histoire des sciences humaines, 17, pp. 4759. 
Nysten P-H., Littré É., Robin C. (1865), " Sexualité », Dictionnaire de médecine, de chirurgie, de pharmacie, des sciences accessoires et de l'art vétérinaire, Nysten P.-H., 12e édition, Paris : Baillière, p. 1381.

Oakley A. (1972), Sex, Gender and Society, London : Temple Smith.

Oosterhuis H. (2000), Stepchildren of nature. Krafft-Ebing, Psychiatry and the Making of Sexual Identity, Chicago : University of Chicago Press.

Raffalovich M. A. (1896), Uranisme et unisexualité. Études sur différentes manifestations de l'instinct sexuel, Paris : Masson.

Revenin R. (2005), Homosexualité et prostitution masculines à Paris. 1870-1918, Paris: L'Harmattan.

Revenin R. (2007), « Conceptions et théories savantes de l'homosexualité en France, de la monarchie de Juillet à la Première Guerre mondiale », Revue d'histoire des sciences humaines, 17, pp. 23-45.

Rey M. (1982), " Police et sodomie à paris au XVIII ${ }^{\mathrm{e}}$ siècle: du péché au désordre ", Revue d'histoire moderne et contemporaine, 29, pp. 113-124.

Rosario V. (2000[1997]), L'irrésistible ascension du pervers, entre littérature et psychiatrie, Paris, EPEL, traduction par Le Gaufey G, de Rosario V. (1997), The Erotic Imagination. French Histories of Perversity, New York : Oxford University Press.

Sibalis M. D. (1996), "The regulation of male homosexuality in Revolutionary and Napoleonic France. 1789-1815 », in Merrick J., Ragan B. T. (éd.), Homosexuality in Modern France, New York : Oxford University Press, pp. 80-101.

Singy P. (2014), L'usage du sexe. Lettres au Dr Tissot, auteur de L'Onanisme (1760), Lausanne: BHMS.

Symonds J. A. (1975[1883]), "A problem in greek ethics, being an inquiry into the phenomenon of sexual inversion », in Havelock . H. (1975[1897]), Sexual inversion, New York : Arno Press, pp. 163-257.

Tamagne F. (2001), Mauvais genre. Une histoire des représentations de l'homosexualité, Paris : La Martinière.

Tamassia A. (1878), "Sull'inversione dell'istinto sessuale », Rivista Sperimentale di Freniatria, vol. 4, pp. 97-117.

Tardieu A. (1859[1857]), Étude médico-légale sur les attentats aux mœurs, 3e édition, Paris : Baillière.

Tissot S. A. (1991[1761]), L'onanisme. Dissertation sur les maladies produites par la masturbation. Nouvelle édition, revue et augmentée de la traduction des citations latines, Paris : La Différence. 
Trumbach R. (1998), Sex and the gender Revolution. Vol. 1. Heterosexuality and the Third Gender in Enlightenment London, Chicago : University of Chicago Press.

Ulrichs K. H. (1994 [1864-1880]), The Riddle of 'Man-Manly' Love. The Pionnering Work on Male Homosexuality, 2 vol., New York: Prometheus Books, traduction de l'allemand par Michael A. Lombardi-Nash.

Vidal Fernando, 2004, "Onanism, Enlightenment Medicine, and the Immanent Justice of Nature ", in Daston, Lorraine et Vidal Fernando (éd.), The moral authority of nature, London : University of Chicago Press, pp. 254-281.

Virey J-J. (1818), "Libertinage », Dictionnaire des Sciences médicales, vol.28, Paris: Pancoucke, pp. 112-155.

Westphal C. (1969), «Die conträre Sexualempfindung. Symptom eines neuropatischen (psychopatischen) Zustandes », Archiv für Psychiatrie und Nervenkrankenheiten, vol.2, pp. 73-108. 